\title{
Modification of equine sperm chromatin decondensation method to use fluorescence in situ hybridization (FISH)
}

\author{
Monika Bugno-Poniewierska1, Zofia Jabłońska², Ewa Stota ${ }^{1}$ \\ ${ }^{1}$ Department of Immuno- and Cytogenetics, National Research Institute of Animal Production, Balice $\mathrm{n}$. \\ Kraków, Poland \\ ${ }^{2}$ Faculty of Biology and Earth Sciences, Jagiellonian University of Krakow Poland
}

\begin{abstract}
Fluorescence in situ hybridization (FISH) is widely used in the study of chromosome structure and organization. Cytogenetic evaluation of chromosomes using FISH technique plays an increasingly important role in diagnosing karyotype changes in both somatic and reproductive cells. The aim of the study was to optimize the conditions of stallion sperm decondensation, which have a significant effect on the results of fluorescence in situ hybridization. Appropriate type and time of decondensation was chosen for the sperm of every stallion. It was found that decondensation performed using a preparation incubated in DTT solution for 1.5 minutes and in SDS solution for 10 seconds proved effective for stallions no. 1 and 2 . An alternative decondensation method performed in an Eppendorf tube, with incubation in DTT solution for 1 minute and in SDS solution for 5 seconds proved effective for stallions no. 3 and 4. Decondensation using DTT and papain solution, a method successfully used for bull spermatozoa, proved inadequate for horse spermatozoa.
\end{abstract}

Key words: stallion, sperm decondensation, FISH technique

\section{Introduction}

Fluorescence in situ hybridization (FISH) has been introduced to improve fertility evaluation technique in stallions. This technique makes it possible to obtain cytogenetic information on chromosomes as well as molecular information concerning DNA structure. The simultaneous hybridization of two or more chromosome-specific probes to spermatozoa and subsequent detection of the signal probes using different fluorescent detection systems enables two or more chromosomes to be localized simultaneously in the same sperm cell and provides a technique providing reasonable estimates of aneuploidy..FISH can be rapidly applied to large numbers of spermatozoa.

Only a few successful studies using the latter technique have been reported in farm animals such as cattle [2,5,8,16-17], pigs [1,14-15] and horses [3].

The small number of studies on stallion semen may be due to methodological problems associated with sperm decondensation in this species. Therefore,

Correspondence: M. Bugno, Dept. of Immuno- and Cytogenetics, National Research Institute of Animal Production, Krakowska 1, 32-083 Balice, Poland; tel.: (+4812) 2588313, fax.: (+4812) 2856033, e-mail: mbugno@izoo.krakow.pl the aim of the study was to optimize the conditions of stallion sperm decondensation, which have a significant effect on the results of fluorescence in situ hybridization.

\section{Materials and methods}

Semen collected from four stallions was stored at $-20 \mathrm{C}$, thawed at room temperature, diluted 5 times with physiological saline $(0.9 \%$ $\mathrm{NaCl}$ ) and delicately mixed for 3 minutes. Diluted semen was centrifuged for 5 minutes at $3000 \mathrm{rev} . / \mathrm{min}$. The supernatant was removed and $50 \mu 1$ of $0.9 \% \mathrm{NaCl}$ was added. The suspension obtained was thoroughly mixed using a pipette.

$10 \mu 1$ of ejaculate was then placed on a defatted glass slide and spread over the whole surface using a second slide. These preparations were dried vertically at room temperature. Sperm concentration on the slide was examined using contrast-phase microscopy. When sperm density was unsatisfactory, the ejaculate was diluted or concentrated by centrifugation to an appropriate concentration. Selected preparations were then dehydrated overnight in ethyl alcohol (95-99.5\%) at $4^{\circ} \mathrm{C}$ and left to dry at room temperature.

Type I decondensation. Semen preparations were incubated in DTT papain solution (1.25 g papain $+0.16 \mathrm{~g}$ DTT $+0.5 \mathrm{ml}$ dimethyl sulfoxide $+0.2 \mathrm{M}$ buffer TRIS to $100 \mathrm{ml}$ ) at $40^{\circ} \mathrm{C}$ for $1 \mathrm{~h}$, and rinsed twice for $2 \mathrm{~min}$ in $0.2 \mathrm{M}$ buffer TRIS, $\mathrm{pH}=8.6$ at room temperature. Dehydratation was carried out in an ascending alcohol series $(95 \%, 97 \%, 100 \%)$ at room temperature for $3 \mathrm{~min}$ and dried vertically. Contrast-phase microscopy was then used to check the degree of sperm decondensation over the whole slide surface. 
Where the degree of sperm decondensation was insufficient, the whole procedure was repeated. Several preparations were chosen and the best area for hybridization was marked.

Type II decondensation. Sperm preparations on which sperm remained undecondensed after type I decondensation was used, were subjected to an alternative decondensation method with our own modifications.

After type I decondensation, stallion sperm preparations were dehydrated in ethyl alcohol (95-99.5\%) for 5 min. $10 \mu$ of $0.25 \mathrm{M}$ DTT suspended in $0.01 \mathrm{M}$ TRIS $+0.9 \% \mathrm{NaCl}$ was placed on each preparation and incubated for $1.5 \mathrm{~min}$ under a cover glass, after which $10 \mu \mathrm{l}$ of the $1 \%$ sodium dodecyl sulfate $+1.9 \%$ disodium tetraborate mixture was dropped on distilled water and also incubated for 5-10 s under a cover glass. Preparations were then immersed in $100 \%$ ethyl alcohol for 5 min and dried at room temperature. The degree of sperm decondensation was checked using contrast-phase microscopy and the appropriate area for hybridization was chosen.

Type III decondensation according to the Cambio protocol. Thawed stallion sperm $(50 \mu \mathrm{l})$ was placed in a $1.5 \mathrm{ml}$ Eppendorf tube and $100 \mu \mathrm{l}$ of $0.01 \mathrm{M}$ TRIS in $0.9 \% \mathrm{NaCl}$ was added. The suspension was centrifuged $(10 \mathrm{~min} / 800 \mathrm{rpm})$, the supernatant was removed and $50 \mu \mathrm{l}$ of $0.01 \mathrm{M}$ TRIS in $0.9 \% \mathrm{NaCl}$ was again added. Washed sperm $(5 \mu \mathrm{l})$ was transferred to a $1.5 \mathrm{ml}$ Eppendorf tube and $5 \mu 1$ of $0.25 \mathrm{M}$ DTT (suspended in $0.01 \mathrm{M}$ TRIS + $0.9 \% \mathrm{NaCl}$ ) was added and incubated for $1 \mathrm{~min}$, after which $5 \mu \mathrm{l}$ of SDS solution ( $1 \%$ sodium dodecyl sulfate $+1.9 \%$ disodium tetraborate in distilled water) was added and again incubated for $5 \mathrm{~s}$. After this time $50 \mu \mathrm{l}$ of $100 \%$ ethanol was added. $10 \mu \mathrm{l}$ of decondensed sperm were then transferred to a defatted slide and spread over the whole surface using a second slide. These preparations were dried vertically at room temperature. Dry preparations were dehydrated in $100 \%$ ethanol for $5 \mathrm{~min}$ and dried again. The degree of sperm decondensation was checked using contrastphase microscopy and the best area for hybridization on the preparation was chosen.

Hybridization. Preparations with decondensed sperm were dehydrated in $100 \%$ ethyl alcohol for $5 \mathrm{~min}$ at room temperature, left to dry and subjected to pepsin digestion at $37^{\circ} \mathrm{C}$ for $30 \mathrm{~min}$. After this time, preparations were washed three times for $1 \mathrm{~min}$ in $2 \mathrm{xSSC}$ buffer at room temperature, and then again washed in distilled water for $3 \mathrm{~s}$. Preparations were then dehydrated in ethyl alcohol series $(70 \%, 80 \%, 95 \%), 2$ min per each concentration. Dehydration was repeated in $100 \%$ ethanol for 4 min, after which preparations were heated at $65^{\circ} \mathrm{C}$ for $30 \mathrm{~min}$ in a thermocycler.

Probes were denatured during digestion of preparations with pepsin. Sex chromosome probes: X - labelled with FITC and Y labelled with $\mathrm{Cy} 3$ were denatured at $70^{\circ} \mathrm{C}$ for $10 \mathrm{~min}$, and $\mathrm{BAC}$ probe of the EGFR gene labelled with digoxygenin was denatured at $80^{\circ} \mathrm{C}$ for $8 \mathrm{~min}$. Immediately after denaturation, the probes were transferred to an incubator at $37^{\circ} \mathrm{C}$ for $30-60 \mathrm{~min}$. Denaturations of sperm preparations were performed in $70 \%$ formamide in $2 x S S C$ at $78^{\circ} \mathrm{C}$ for $10 \mathrm{~min}$. Immediately after denaturation, preparations were immersed in ice-cold $70 \%$ ethanol chilled to $-20^{\circ} \mathrm{C}$, and then passed through an ice-cold alcohol series $(80 \%, 90 \%, 100 \%)$ and left to dry at room temperature. After preannealing, the probes were combined and $5 \mu 1$ of each was placed on denatured preparations, which were then covered with a cover glass and edges of the preparation were sealed with Fixogum. Hybrydization was carried out in a moist chamber at $37^{\circ} \mathrm{C}$ for 3 days.

Posthybridization. After hybridization, preparations were soaked in 2xSSC buffer, cover glass was removed and several washes were performed, twice for $5 \mathrm{~min}$ in $50 \%$ formamide in $2 \mathrm{xSSC}$ and in $2 \mathrm{xSSC}$ buffer alone at $43^{\circ} \mathrm{C}$. Preparations were next placed in
Detergent Wash Solution $(100 \mathrm{ml} 20 \mathrm{xSSC}+400 \mathrm{ml}$ distilled water $+250 \mu 1$ Tween 20$)$ at $43^{\circ} \mathrm{C}$ for $4 \mathrm{~min}$.

Cambio Detection Kit was used to detect fluorescent signal in sperm. After the preparations were incubated in Detergent Wash Solution, $50 \mu 1$ of working solution D was placed on every preparation and covered with a cover glass, after which preparations were incubated in a moist chamber for $20 \mathrm{~min}$ at $37^{\circ} \mathrm{C}$. At the end of incubation, preparations were washed three times in Detergent Wash Solution for $4 \mathrm{~min}$ at room temperature. $200 \mu \mathrm{l}$ of Anti Digoxigenin Rhodamine (suspended in PBS $+0.5 \%$ BSA, $\mathrm{pH}=$ 7.4) was then placed on each preparation and incubated in a humidified chamber for 30 at $37^{\circ} \mathrm{C}$, followed by repeated washing in Detergent Wash Solution three times. After washing, preparations were drained and $10 \mu \mathrm{l}$ of DAPI (4'-6-Diamidino-2-phenylindole) was placed on each preparation, after which they were covered with a cover glass.

\section{Results}

Appropriate type and time of decondensation was chosen individually for each stallion. The first decondensation method using DTT and papain solution, which is successfully used on bull spermatozoa [2] proved inadequate for horse spermatozoa. The second decondensation method, performed on a glass slide, with incubation in DTT solution for $1.5 \mathrm{~min}$ and in SDS solution for $10 \mathrm{~s}$ proved efficient for stallion no. 1 and 2. The third decondensation type, performed in an Eppendorf tube, with incubation in DTT solution for 1 min and in SDS solution for $5 \mathrm{~s}$ was efficient for stallion no. 3 and 4. Successive stages of sperm decondensation are shown in Fig. 1.

After using FISH technique on uncondensed sperm, no fluorescent signals are observed due to the lack of hybridization between molecular probe and sperm chromatin, whereas fluorescent signals on overcondensed sperm are scattered and unclear (Fig. 2).

Where sperm chromatin decondensation is normal, fluorescent signals of molecular probes are clear and easy to analyse, regardless of the size of the probe used (probes painting whole chromosomes or gene-specific probes; Fig. 3).

\section{Discussion}

The first attempts to use FISH technique in spermatozoa showed that it is necessary to use preliminary decondensation of sperm nucleus to enable hybridization between DNA probe and appropriate site on sperm chromatin [12]. In the present study, differences were observed in the degree of decondensation between individual stallions and appropriate type and duration of decondensation was chosen for each animal analysed. Sperm chromatin decondensation was obtained due to:

- enzymatic properties of papain that digests the peptide bond between lysine and arginine,

- sodium dodecyl sulfate (SDS), which is an anionic, lysing ionic detergent that causes pro- 

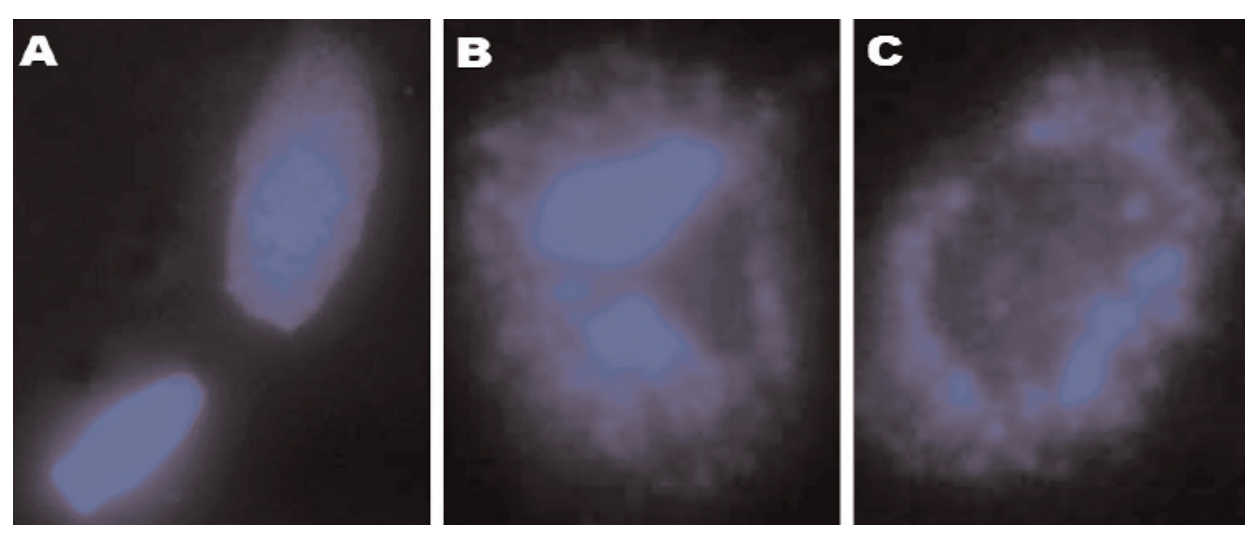

Fig. 1. Sample degrees of stallion sperm decondensation, DAPI staining. (A) uncondensed sperm (bottom) and condensed sperm (top), (B) overcondensed sperm, (C) completely overcondensed sperm.

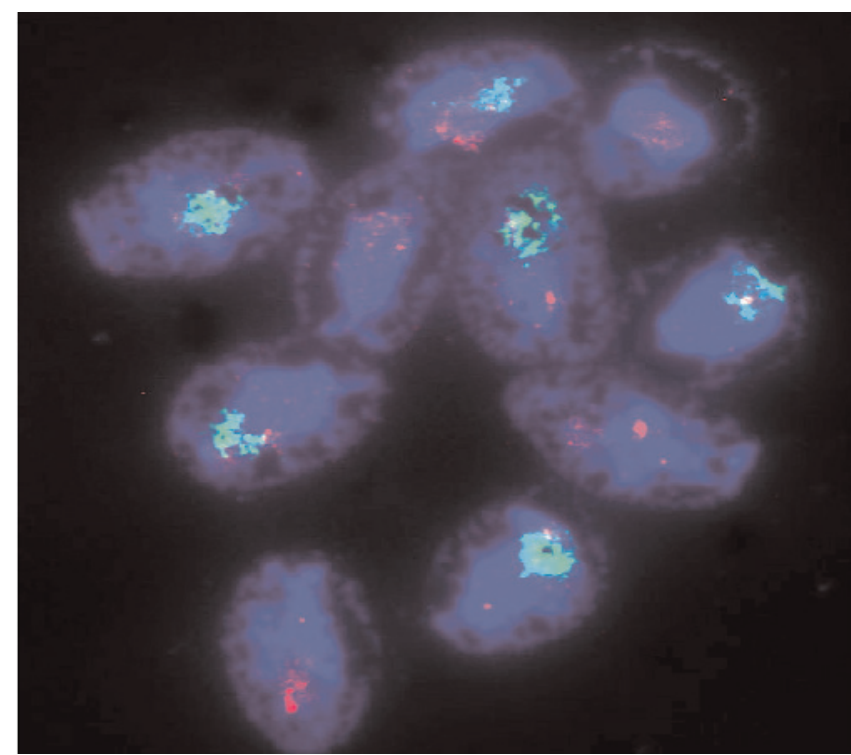

Fig. 2. Highly condensed sperm with fragmentary and unclear signal after using FISH technique.

tein denaturation, dissociation of subunits (by breaking non-covalent bonds) and SDS coating while inhibiting ribonuclease activity (due to protein denaturation),

- dithiothreitol (DTT), which is a strong agent that reduces disulfide bridges and prevents the formation of intra- and intermolecular bridges between residues in proteins, thus destroying their tertiary structure. The reducing capacity of DTT is limited to $\mathrm{pH}$ values above 7 . For this reason TRIS, which is a buffer solution maintaining stable (slightly alkaline) $\mathrm{pH}$ was used,

- the detergent disodium tetraborate, which dissolves lipids.

Differences within series and between animals or species may result from the number of disulfide bonds in the sperm nucleus $[9,13]$. Bull spermatozoa are condensed more easily than stallion spermatozoa because bull sperm nuclei are characterized by a higher concentration of disulfide bonds compared to human or
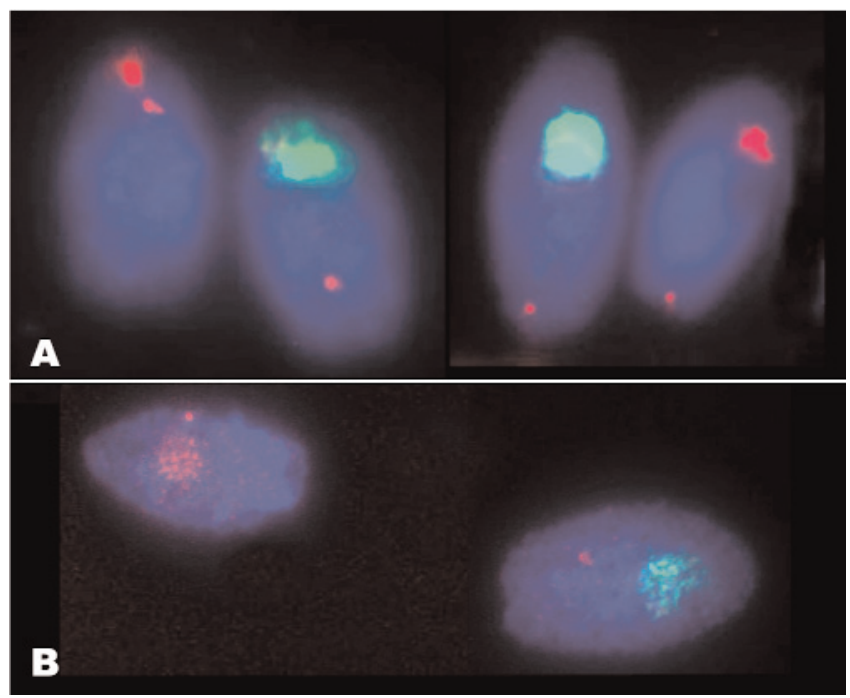

Fig. 3. (A) Normal sperm containing $X$ chromosome (green signal) and Y chromosome (red signal) as well as EGFR gene (small red signal). (B) slightly overcondensed sperm, with a scattered but still clear signal.

mouse sperm nuclei and therefore they seem more resistant to the action of chemical preparations [9].

For decondensation of sperm heads in pigs, to detect chromosome $\mathrm{Y}$ carrying sperm using in situ hybridization, 2mM DTT was used [11]. In humans, sperm decondensation was successful using DTT and lithium diiodosalicylate with salts $[6-7,10,18]$. In humans and in pigs, sperm nuclei were also successfully decondensed using glutathione (GSH) $[9,11]$, but this reagent proved inefficient for bull spermatozoa [12]. Glutathione is the major intercellular free thiol in mammalian oocytes. It plays a major role in reducing disulfide bonds between protamine, which contributes to subsequent dispersion of sperm chromatin during fertilization [13]. The use of GSH combined with heparin produced the expected result of decondensation of bull sperm nucleus [12]. Heparin shows strong affinity for protamine molecules [4]. Sperm nucleus condensation results from the formation of protamineDNA complexes linked by disulfide bridges, which 
means that the increased sperm head was probably due to heparin because it competes with DNA for binding protamine molecules [12].

Acknowledgements: This work was financed by the National Research Institute of Animal Production, project No 3214.1 and NN311 310636.

\section{References}

[ 1] Benkhalifa M, Janny L, Malet P, Boucher D, Menezo Y, Vye P, Pouly JL. Chromosome studies of human gametes and embryos by fluorescent in situ hybridization. Hum Reprod. 1992;7:68-69.

[ 2] Bugno M, Jabłońska Z, Słota E. Double colour fluorescence in situ hybridisation with $\mathrm{X}$ and $\mathrm{Y}$ painting probes in bovine spermatozoa. Ann Anim Sci. 2008; in press.

[ 3] Bugno M, Jabłońska Z, Tischner M, Słota E. Detection of sex chromosomem and estimation of aneuploidy in horse spermatozoa using fluorescence in situ hybridisation (FISH). $7^{\text {th }}$ Inernational Symposium on Equine Embryo Transfer. Cambridge 9-11 July 2008; 2008: pp. 34-35.

[4] Chargaff E, Olson KB. Studies on the chemistry of blood anticoagulation. VI. Studies on the action of heparin and other coagulants. The influence of protamine on the anticoaglulant effect in vivo. J Biol Chem. 1936;122:153-1 67.

[5] Di Berardino D, Vozdova M, Kubickova S, Cernohorska H, Coppola G, Coppola G, Enne G, Rubes J. Sexing river buffalo (Bubalus bubalis L.) sheep (Ovis aries L.), goat (Capra hircus L.) and cattle spermatozoa by double color FISH using Bovine (Bos Taurus L.) X- and Y-painting probes. Mol Reprod Dev. 2004;67:108-115.

[ 6] Han TL, Flaherty SP, Ford JH, Matthews CW. Detection of Xand Y-bearing human spermatozoa after motile sperm isolation by swim-up. Fertil Steril. 1993;60:1046-1051.

[7] Han TL, Webb GC, Flaherty SP, Correll A, Matthews CD, Ford JH. Detection of chromosome 17- and X-bearing human spermatozoa using fluorescence in situ hybridization. Mol Reprod Dev. 1992;33:169-1 94.

[ 8] Hassanane M, Kovacs A, Pascal L, Lindblad K, Gustavsson I. Simultaneous detection of X- and Y-bearing bull spermatozoa by double colour fluorescence in situ hybridization. $\mathrm{Mol}$ Reprod Dev. 1999;53:407-412.

[ 9] Jager S, Wijchman J, Kremer J. Study on the decondensation of human, mouse, and bull sperm nuclei by heparin and other polyanions. J Exp Zool. 1990;256:315-322.

[10] Joseph AM, Gosden JR, Chandly AC. Estimation of aneuploidy levels in human spermatozoa using chromosome specific probes and in situ hybridization. Hum Genet. 1984;66: 234-238.

[11] Kawarasaki T, Kohsaka T, Sone M, Yoshida M, Bamba K. Detection of Y-bearing porcine spermatozoa by in situ hybridization using digoxigenin-labeled, porcine male-specific DNA probe produced by polymerase chain reaction. $\mathrm{Mol}$ Reprod Dev. 1995;40:455-459.

[12] Kobayashi J, Kohsaka T, Sasada H, Umezu M, Sato E. Fluorescence in situ hybrydization with chromosome-specific probe in decondensed spermatozoa. Theriogenology. 1999; 52:1043-1054.

[13] Perreault SD, Barbee RR, Elstein KH, Zucker RM, Keefer CL. Intrespecies differences in the stability of mammalian sperm nuclei assessed in vivo by sperm microinjection and in vitro by flow cytometry. Biol Reprod. 1988;39:157-167.

[14] Pinton A, Ducos A, Yerle M. Estimation of the proportion of genetically unbalanced spermatozoa in the semen of boars carrying chromosomal rearrangements using FISH on sperm nuclei. Genet Sel Evol. 2004;36:123-137.

[15] Pinton A, Raymond Letron I, Berland HM, Bonnet N, Calgaro A, Garnier-Bonnet A, Yerle M, Ducos A. Meiotic studies in an azoospermic board carrying a Y;14 translocation. Cytogenet. Genome Res. 2008;120:106-111.

[16] Tateno H, Miyake YI, Mori H, Kamiguchi Y, Mikamo K. Sperm chromosome study of two bulls heterozygous for different Robertsonian translocations. Hereditas. 1994;120:7-11.

[17] Vozdova M, Kubickova S, Cernohorska H, Rubes J. Detection of translocation rob $(1 ; 29)$ in bull sperm using a specific DNA probe. Cytogenet Genome Res. 2008;120:102-105.

[18] Wyrobek AJ, Alhborn T, Balhorn R, Stanker L, Pinkel D. Fluorescence in situ hybridization to Y chromosomes in decondensed human sperm nuclei. Mol Reprod Dev. 1990;27:200208.

Submitted: 4 September, 2009 Accepted after reveiws: 26 November, 2009 